Full Length Article

\title{
Towards application of a covalent organic framework-silver nanoparticles@sand heterostructure as a high-efficiency catalyst for flow-through reduction of organic pollutants
}

\author{
Fei Pan ${ }^{\mathrm{a}, \mathrm{c}}$, Fengyan Xiao ${ }^{\mathrm{b}}$, Ning Wang ${ }^{\mathrm{a}, \mathrm{d}, \text { * }}$ \\ ${ }^{a}$ CAS Key Laboratory of Coastal Environmental Processes and Ecological Remediation, Shandong Key Laboratory of Coastal Environmental Processes, YICCAS; Research \\ Center for Coastal Environmental Engineering and Technology of Shandong Province, Yantai Institute of Coastal Zone Research, Chinese Academy of Sciences, Yantai, \\ Shandong Province 264003, PR China \\ ${ }^{\mathrm{b}}$ Yantai Vocational College, Yantai, Shandong Province 264074, PR China \\ ${ }^{\mathrm{c}}$ University of Chinese Academy of Sciences, Beijing 100049, PR China \\ ${ }^{\mathrm{d}}$ Center for Ocean Mega-Science, Chinese Academy of Sciences, 7 Nanhai Road, Qingdao 266071, PR China
}

\section{A R T I C L E I N F O}

\section{Keywords:}

Flow-through

Covalent organic frameworks

Organic pollutants

Catalyst

Application

Silver nanoparticles

\begin{abstract}
A B S T R A C T
Noble metal nanoparticles supported by covalent organic frameworks (COFs) exhibited excellent catalytic performances towards organic pollutants, while their applications are limited by the recovery and reuse from the mixture due to their small size. Here, we solved this issue by supporting covalent organic framework-silver nanoparticles on sand and used as a catalyst for reduction of organic pollutants. Both static and flow-through experiments were carried out and good catalytic performances were exhibited. High flux $\left(2000 \mathrm{~L} \mathrm{~m}^{-2} \mathrm{~h}^{-1}\right)$ / reduction efficiency (more than 99\%) towards 4-nitrophenol, Congo red, and methylene blue, were obtained under the flow-through process. Furthermore, after testing $24 \mathrm{~h}$, the reduction efficiency was unaltered and only $0.34 \mathrm{wt} \% \mathrm{Ag}$ ions were released from the catalyst. Besides the excellent cocatalyst of COF and the stable structure of our prepared catalyst, these good catalytic performances can be also attribute to the properties of sand which has a large size to prevent the leaching out of catalysts and a good hydrophilicity for fast passing through of wastewater. Therefore, our prepared catalyst may shed light on the application of such kinds of heterogeneous catalysts for the fast and continuous treatment of toxic and carcinogenic organic pollutants.
\end{abstract}

\section{Introduction}

Heterogeneous catalysts based on covalent organic frameworks supporting noble metal nanoparticles have gained great interest recently due to their fascinated properties for catalytic reduction of organic pollutants [1-6]. The strong anchoring ability between noble metal nanoparticles and functional groups on the skeleton of covalent organic frameworks (COFs) could enhance the stability of COFs-noble metal nanoparticles, thus improving the stability and sustainable ability of these kinds of catalysts [7]. Nevertheless, the application of these COFsnoble metal nanoparticles catalysts was limited by the continuous flow catalytic process. On the one hand, the continuous compression of feed solution towards the stacked COFs forms a dense structure under the flow-through process, leading to the heavily decreasing of flux [8]. On the other hand, the small-sized catalysts would leach out during the flow process, increasing the cost and difficulty of separation [6]. Considered the characteristics of the flow-through catalytic process, it is necessary to select a suitable substrate to load COFs-noble metal nanoparticles for continuous catalytic application.

Sand, a natural extreme low-cost material, has the advantages of loosely stacked pore structures and good hydrophilicity, facilitating the fast passing through the feed solution and continuous catalytic reduction of organic pollutants. Besides, the large-sized sand is stable and difficult to leach out during the continuous-flow process. However, the usage of sand as a substrate to support catalysts for organic pollutants removal draws less attention.

Covalent organic frameworks-silver nanocomposites supported by sand (COFs-Ag NPs@Sand) were fabricated here. COF with a large surface area was synthesized under high temperature for abundant supporting noble metal nanoparticles. Ag NPs were selected because of their high cost-effectiveness for organic pollutants removal [9-11]. 4nitrophenol, Congo red, and methylene blue were chosen as model

\footnotetext{
* Corresponding author.

E-mail address: nwang@yic.ac.cn (N. Wang).
} 
a<smiles>Nc1ccc(-c2cc(-c3ccc(N)cc3)cc(-c3ccc(N)cc3)c2)cc1</smiles><smiles>C=Cc1cc(C=O)c(C=O)cc1C=O</smiles>

\section{1, 2-dichlorobenzene n-butanol}

$120^{\circ} \mathrm{C} 3$ days

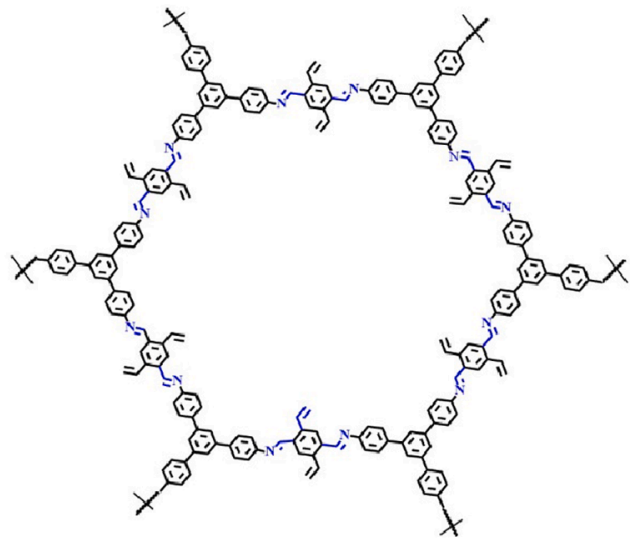
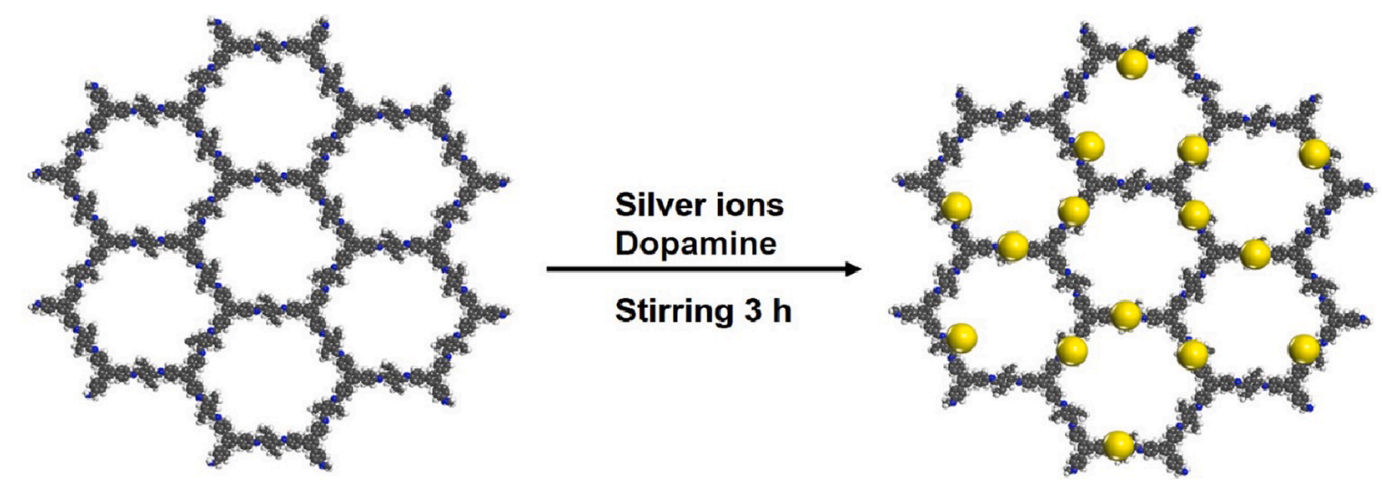

c

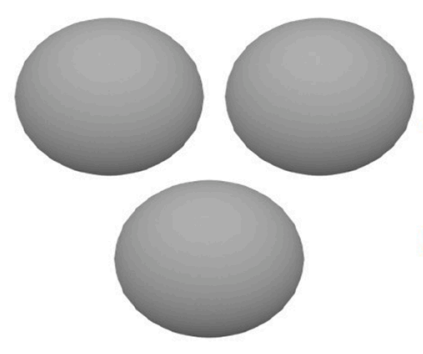

Sand

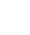

1. Dopamine

2. $\mathrm{COF} @ \mathrm{AgNO}_{3}$
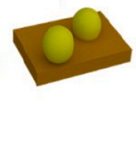

\section{COF-Ag NPs@Sand}

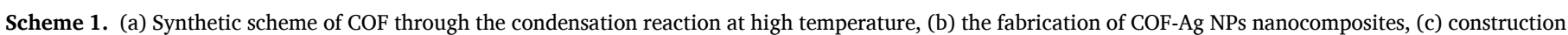

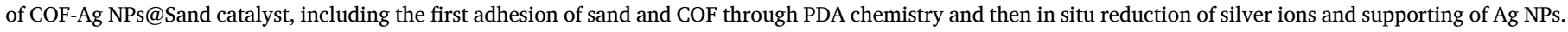

organic pollutants because their high toxic and classic catalytic treatment process [12-14]. For example, through the hydrogenation reaction under the noble metal catalyst, the carcinogenic 4-nitrophenol (4-NP) can be converted to 4-aminophenol (4-AP), which can be efficiently collected by cationic resin and reuse as an industrial intermediate [15-19]. The catalytic performances towards three model organic pollutants in the presence of $\mathrm{NaBH}_{4}$ were systematically evaluated under the static and continuous flow-through catalytic process. As far as we know, this is a rare study to explore the fast-continuous flow-through catalytic process using a natural material supporting COF-Ag NPs catalytic system. Such a strategy provides a guideline for designing the fabrication of high-efficiency catalysts for sustainable organic pollutants treatment.

\section{Experimental section}

\subsection{Materials and methods}

The reagents including 1, 3, 5 - Tris (4-aminophenyl) benzene ( $\geq 98.0 \%), 2$, 5-divinylterephthalaldehyde $(\geq 97.0 \%)$, and dopamine hydrochloride $(\geq 98.0 \%)$ were obtained from Acros. The reagents including 4-nitrophenol (4-NP, $\geq 98.0 \%$ ), methylene blue (MB, $\geq$ 
a

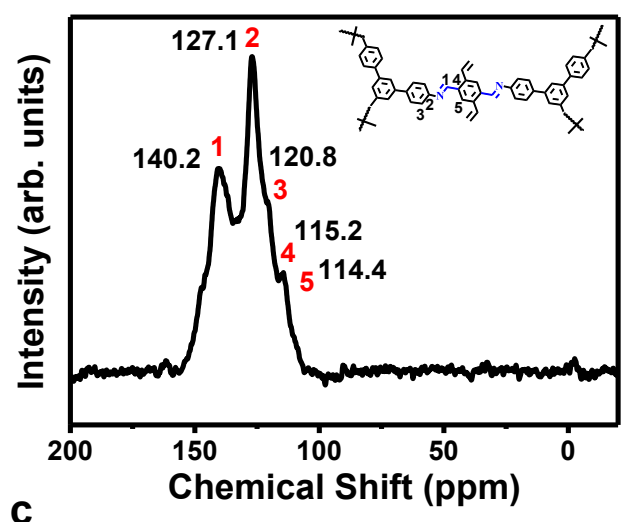

C

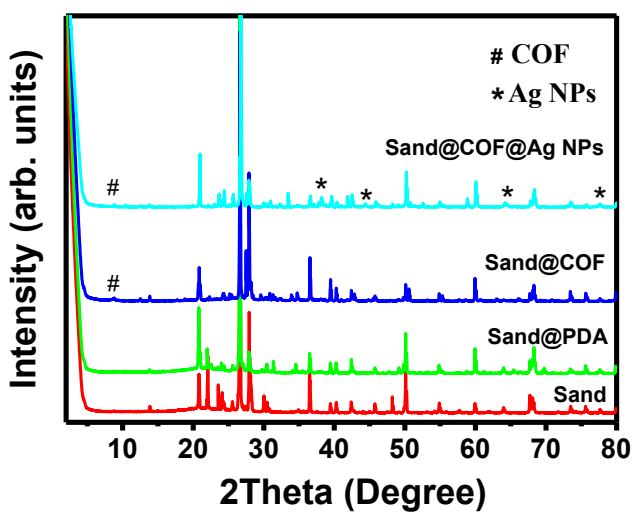

b

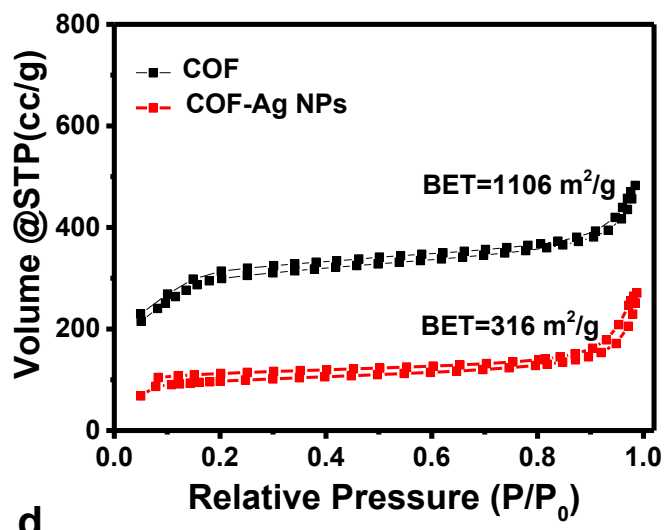

d

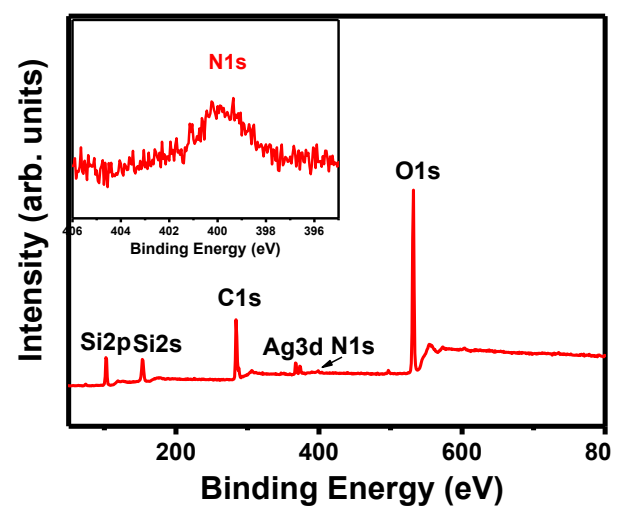

Fig. 1. Structural study of samples through (a) solid-state NMR, (b) BET measurement, (c) XRD, and (d) XPS with inset of N1s of COF-Ag NPs@Sand catalyst.

98.0\%), and Cong red (CR, $\geq 98.0 \%)$ were purchased from Tianjin Guangfu Reagent Co., China. Other reagents including sodium borohydride $\left(\mathrm{NaBH}_{4}, \geq 99.0 \%\right), 1,2$-dichlorobenzene ( $\geq 97.0 \%$ ), and n-butanol were supplied by Sinopharm Chemical Reagent Co. Ltd., China. Sea sand was picked up from the Yantai coast, China, and used after washing with DI water and filtering through the 50-mesh size. The morphologies of the samples were observed by scanning electron microscopy (SEM, Jeol S4800) and transmission electron microscopy (TEM, JEM 2200). The composition was performed with TEM coupled with an energydispersive detector and X-ray photoelectron spectroscopy (XPS, Thermo EscaLab 250Xi). The catalytic performances were systematically evaluated by a UV-visible (UV-vis) spectrometer (PERSEE UT 1810).

\subsection{Synthesis of $\mathrm{COF}$}

2, 5-divinylterephthalaldehyde $(0.48 \mathrm{mmol})$ and $1,3,5$ - Tris (4aminophenyl) benzene $(0.32 \mathrm{mmol})$ were weighed into a Schlenk tube and dissolved in $4.0 \mathrm{~mL}$ mixed solution containing $2.0 \mathrm{~mL} \mathrm{n}$-butanol, 0.4 $\mathrm{mL} 6 \mathrm{M}$ acetic acid, and $2.0 \mathrm{~mL} \mathrm{1,2-dichlorobenzene.} \mathrm{Three} \mathrm{successive}$ freeze-pumpthaw cycles were carried out and then the reaction was maintained at $100{ }^{\circ} \mathrm{C}$ for $72 \mathrm{~h}$. The obtained yellow-brown precipitate was first collected through filtration, followed by washing with tetrahydrofuran by Soxhlet extraction for $48 \mathrm{~h}$. Finally, the product was dried under vacuum at $70{ }^{\circ} \mathrm{C}$ for $24 \mathrm{~h}$ to obtain COF.

\subsection{Synthesis of COF-Ag NPs@Sand}

$10 \mathrm{mM}$ Tris- $\mathrm{HCl}$ buffer was added into a beaker containing $5.0 \mathrm{~g}$ of sand and $0.5 \mathrm{~g}$ of dopamine hydrochloride and reacted $6 \mathrm{~h}$ under stirring. Then the black product (polydopamine modified sand, PDA@Sand) was fully washed with $25 \%$ isopropyl alcohol aqueous solution and dried under a vacuum oven at $70^{\circ} \mathrm{C}$ for $24 \mathrm{~h}$. On the other hand, $200 \mathrm{mg}$ of synthesized COF was added in $200 \mathrm{~mL}$ DI water and suffered ultrasonic treatment for $3 \mathrm{~h}$ via a cell disruptor to get the dispersed yellow suspension. Then $0.34 \mathrm{~g}$ of $\mathrm{AgNO}_{3}$ was added and further reacted for $3 \mathrm{~h}$. After that, $2 \mathrm{mg}$ of dopamine hydrochloride was added into the mixture and reacted for $3 \mathrm{~h}$. Then the products (COF-Ag NPs) were washed with DI water for 3 times and then dried under a vacuum oven at $70{ }^{\circ} \mathrm{C}$ for 24 h. For supporting Ag NPs with different contents on the sand, $0.17 \mathrm{~g}$ and $0.68 \mathrm{~g}$ of $\mathrm{AgNO}_{3}$ was added to the COF system and reacted for $3 \mathrm{~h}$, respectively. $5.0 \mathrm{~g}$ of dried PDA@Sand was added in $300 \mathrm{~mL}$ DI water containing $200 \mathrm{mg}$ of COF-Ag NPs nanocomposites and mixed $6 \mathrm{~h}$ under a shaker. Then the prepared COF-Ag NPs@Sand catalyst was washed with DI water 3 times and then dried under a vacuum oven at $70{ }^{\circ} \mathrm{C}$ for 24 h. Besides, Sand@Ag NPs catalyst was fabricated through PDA chemistry for control. Typically, $0.34 \mathrm{~g}$ of $\mathrm{AgNO}_{3}$ and $5.0 \mathrm{~g}$ of dried PDA@Sand were mixed with $200 \mathrm{~mL}$ and further reacted $1 \mathrm{~h}$ to obtain the product Sand-Ag NPs.

\subsection{Catalytic experiments}

The catalytic performances were evaluated by static and flowthrough experiments. Three organic pollutants were chosen including 4-NP, CR, and MB.

For static experiments, $100 \mathrm{~mL}$ of aqueous solution containing $0.5 \mathrm{~g} /$ $\mathrm{L}$ catalyst, $12 \mathrm{mg} / \mathrm{L}$ 4-NP (25 mg/L for CR and MB), and $0.05 \mathrm{M} \mathrm{NaBH}_{4}$ was monitored by the UV-vis absorption spectra and the timedependent concentration of the mixture was recorded. The apparent reduction rate constant $K_{\text {app }}$ was expressed as follows:

$\ln \left(\frac{\mathrm{C}_{\mathrm{t}}}{\mathrm{C}_{0}}\right)=-\mathrm{K}_{\mathrm{app}} \mathrm{t}$

Where $C_{t}$ is the dyes concentration at the time $t$ and $C_{o}$ are the initial concentration. The value can be obtained from calibration curves 

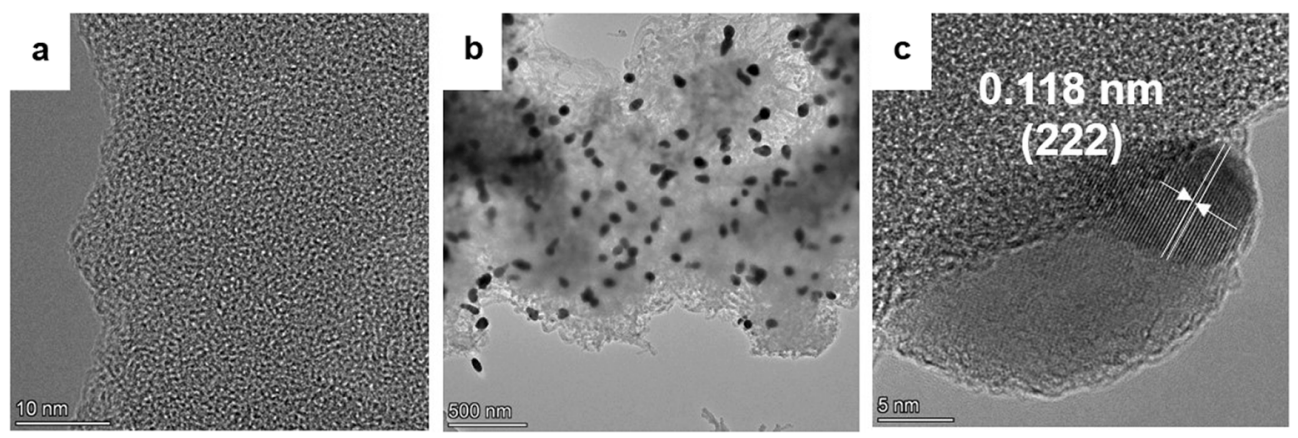

Fig. 2. TEM images of (a) COF, (b) COF-Ag NPs, and (c) COF-Ag NPs@Sand catalyst through high solution TEM.

(Fig. S1).

The recyclability experiments were carried out through pouring away the reaction solution and rejoined the fresh solution containing organic pollutants and $\mathrm{NaBH}_{4}$. No devices were involved due to the good sedimentary properties of sand. The reduction efficiency $(E)$ was calculated according to the following equation:

$E=\frac{C_{0}-C_{t}}{C_{0}} \times 100 \%$

For flow-through experiments, the flux was defined as follows (3).

$H=\frac{V}{S \times t}$

Where $\mathrm{H}$ is the flux, $\mathrm{V}$ is the cumulative permeability volume and $S$ is the cross-section of pipeline.

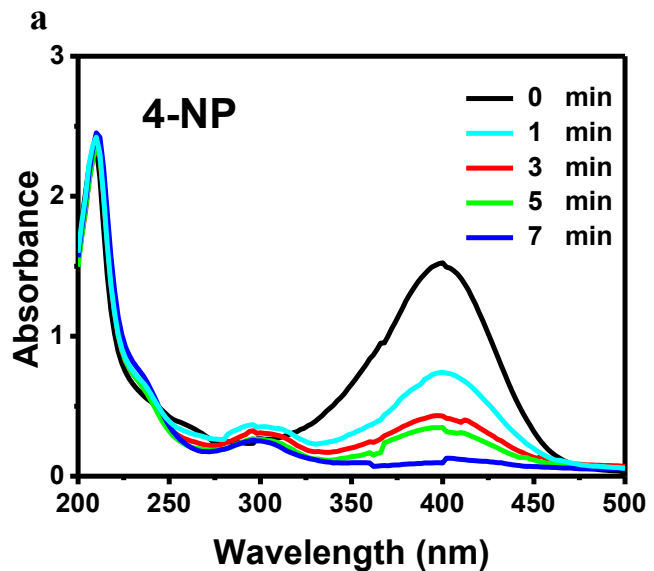

C

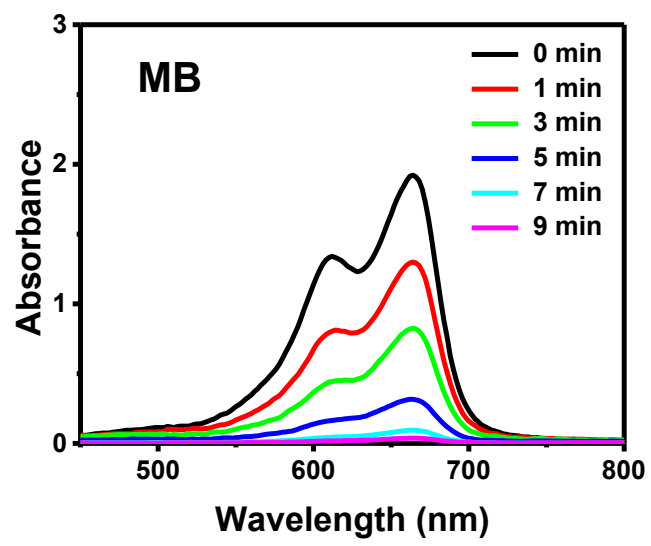

Typically, 400 mg of COF-Ag NPs@Sand catalyst was first loaded into a PVC tube with a diameter of $6 \mathrm{~mm}$ and an effective length of 10 $\mathrm{mm}$ and sealed with cotton. The solution containing $0.05 \mathrm{M} \mathrm{NaBH}_{4}$ and $12 \mathrm{mg} / \mathrm{L}, 40 \mathrm{mg} / \mathrm{L}$, and $25 \mathrm{mg} / \mathrm{L}$ for 4-NP, CR, and MB, respectively, was continuous catalytic reduction by the COF-Ag NPs@Sand catalyst.

\section{Results and discussion}

\subsection{Synthesis and characterization of COF-Ag NPs@Sand catalyst}

A COF was constructed using 1, 3, 5 - Tris (4-aminophenyl) benzene as the knot and 2, 5-divinylterephthalaldehyde as the organic linker through the condensation reaction at high temperature [20]. Then $\mathrm{Ag}$ NPs were in situ growth on the surface of the prepared COF through dopamine chemistry, followed by depositing on the surface of

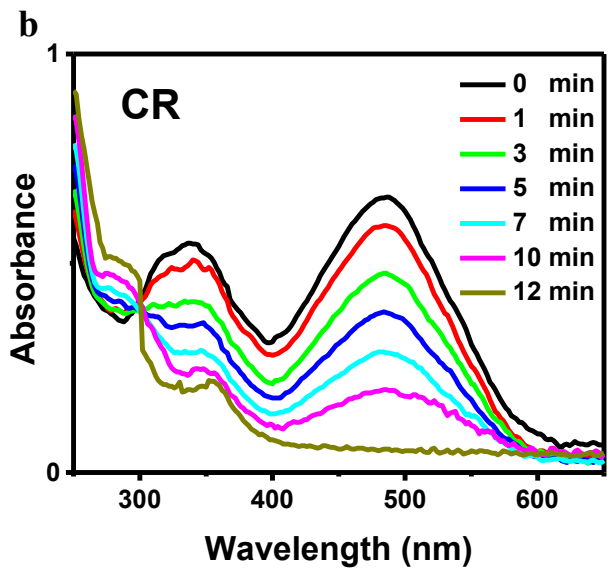

d

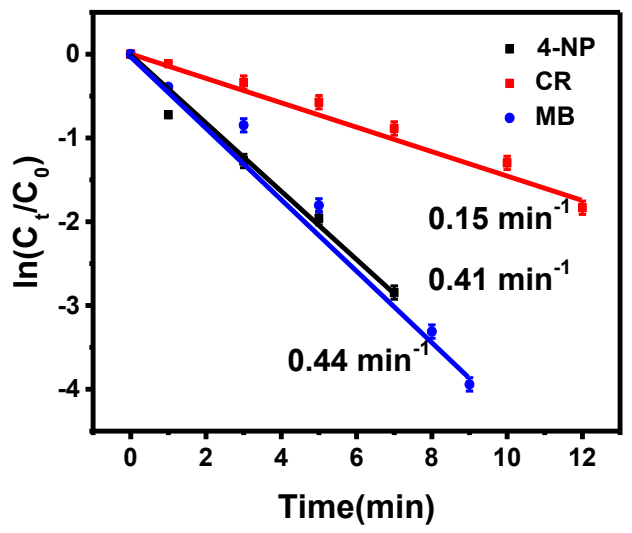

Fig. 3. The time-resolved UV-vis spectra of dyes solutions containing (a) 4-NP $\left(12 \mathrm{mg} \mathrm{L}^{-1}\right)$, (b) $\mathrm{CR}\left(25 \mathrm{mg} \mathrm{L}^{-1}\right)$, and (c) $\mathrm{MB}\left(25 \mathrm{mg} \mathrm{L}^{-1}\right)$ with $\mathrm{NaBH}_{4}\left(0.05 \mathrm{M}^{2}\right)$ and COF-Ag NPs@Sand catalyst $\left(0.5 \mathrm{~g} \mathrm{~L}^{-1}\right)$. (d) Plot of $\ln \left(\mathrm{C}_{\mathrm{t}} / \mathrm{C}_{0}\right)$ versus reaction time for the catalytic reduction of 4-NP, CR, and MB. 

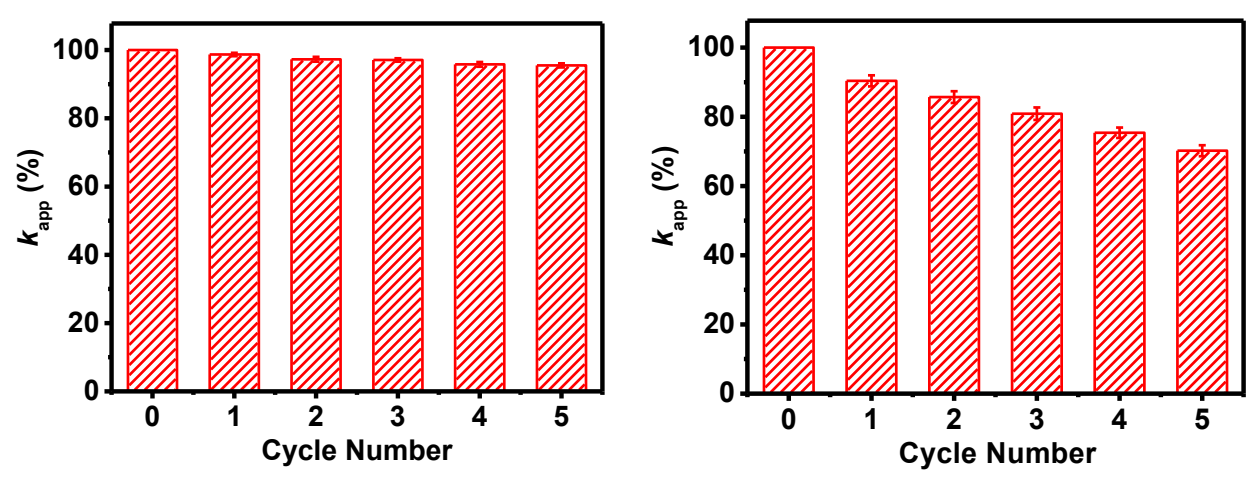

Fig. 4. Recyclability of (a) COF-Ag NPs@Sand catalyst in the reduction of 4-NP (Condition: 4-NP (12 mg L $\left.\mathrm{L}^{-1}\right), \mathrm{NaBH}_{4}(0.05 \mathrm{M})$, and COF-Ag NPs@Sand catalyst (0.5 $\left.\mathrm{g} \mathrm{L}^{-1}\right)$ ) and (b) COF-Ag NPs in the reduction of 4-NP (Condition: 4-NP $\left(12 \mathrm{mg} \mathrm{L}^{-1}\right), \mathrm{NaBH}_{4}(0.05 \mathrm{M})$, and COF-Ag NPs@Sand catalyst (0.018 g L $\left.\mathrm{L}^{-1}\right)$ ).

polydopamine modified sand to form COF-Ag NPs@Sand catalyst (Scheme 1).

\subsection{Structural study}

Solid-state ${ }^{13} \mathrm{C}$ cross-polarization magic angle spinning (CP-MAS) NMR spectroscopy was applied to characterize the formation of $\mathrm{C}=\mathrm{N}$ bonding in the synthesized COF. As shown in Fig. 1a, the strong peak at the chemical shift of $140.2 \mathrm{ppm}$ can be assigned to the linkages of $\mathrm{C}=\mathrm{N}$ [21]. The BET surface areas of the COF and the COF-Ag NPs were characterized by the $\mathrm{N}_{2}$ sorption measurement at $77 \mathrm{~K}$. The value of the COF was calculated to be $1106 \mathrm{~m}^{2} / \mathrm{g}$, providing a large surface area for supporting Ag NPs (Fig. 1b). The loading of Ag NPs decreased the value to $316 \mathrm{~m}^{2} / \mathrm{g}$ due to the changing of the surface property [22]. Nevertheless, the high BET value of COF-Ag NPs still indicated a pore structure after loading of Ag NPs. XRD was conducted to analyze the crystallinity. The characteristics peak of Ag NPs and COF could be observed from the sample of COF-Ag NPs@Sand, suggesting the successful supporting of $\mathrm{COF}$ and $\mathrm{Ag}$ NPs on the surface of the catalyst (Fig. 1c). Three peaks at $2 \theta$ of $5.4^{\circ}, 7.4^{\circ}$, and $22.5^{\circ}$ can be observed from the COF, assigning to (1 10$),(200)$, and (001) diffractions (Fig. S2) [23]. For COF-Ag NPs, typically Ag (0) peaks can be observed [24]. However, the peak of COF cannot be observed probably due to the strong crystalline peak of Ag NPs [6].The XPS spectrum of COF-Ag NPs@Sand revealed the signals of silicon (Si 2 s, $152 \mathrm{eV}$, and Si 2p, $101 \mathrm{eV}$ ), N (N 1 s, $400 \mathrm{eV}$ ), and Ag (Ag 3d, $368 \mathrm{eV}$ ), demonstrating the fabrication of COF-Ag NPs@Sand catalyst (Fig. 1d). Furthermore, the contents of Ag NPs on the surface of COF-Ag NPs and COF-Ag NPs@Sand catalyst were calculated as 3.09 wt $\%$ and $0.11 \mathrm{wt} \%$, respectively, by ICP/MS. Therefore, the weight percent of COF-Ag NPs on the prepared COF-Ag NPs@Sand catalyst was quantified to be $3.55 \mathrm{wt} \%$.

\subsection{Morphological study}

After supporting with COF and Ag NPs, the surface morphology of sand was almost unchanged, exhibiting a smooth surface, as shown in Fig. S3a. From the enlarging image of Fig. S3a, the same particles could be observed after functionalizing with polydopamine. Furthermore, an obvious rough surface could be seen from the enlarging image of Fig. S3b, reflecting the supporting of COF-Ag NPs by sand. To further observe the particle size and distribution of the Ag NPs on the surface of COF-Ag NPs@Sand catalyst, TEM coupled with elemental mapping images was applied. COF with good crystallinity was observed in Fig. 2 a. Ag NPs were stably dispersed on the COF-Ag NPs@Sand catalyst (Fig. 2b). Ultrafine Ag NPs with a diameter of approximately $5 \mathrm{~nm}$ and an inter-planar spacing of $0.118 \mathrm{~nm}$ could be seen from the high solution TEM image (Fig. 2e), which can be assigned to the (222) plane of Ag crystal [25]. Furthermore, the loading of Ag NPs had not altered the crystallinity morphology of COF. Although the content of Ag is only
0.11 wt $\%$ on the prepared COF-Ag NPs@Sand catalyst, the well dispersed Ag NPs could supply largely active sites for organic pollutants removal.

\subsection{Catalytic properties of COF-Ag NPs@Sand}

COFs supported ultrafine noble metal nanoparticles have exhibited excellent catalytic performances towards organic pollutants. Hence, the catalytic activity of COF-Ag NPs supported by sand is expected. Firstly, organic pollutant 4-NP was selected as the model due to its high toxicity and stability $[26,27]$. The transform of 4-NP to less toxic 4-aminophenol (4-AP) through the noble metal catalyst in the presence of $\mathrm{NaBH}_{4}$ is an effective approach to reduce the environmental risk and realize the $r$ euse of hazardous waste [28]. The catalytic process was monitored by UV-vis spectroscopy. As shown in Fig. 3a, the characteristic absorbance at $400 \mathrm{~nm}$ vanished within $7 \mathrm{~min}$, accordingly, the absorbance at 300 $\mathrm{nm}$ occurred, assigning to the peak of 4-AP. The samples of PDA coated sand and Sand@COF catalyst were used as controls. As shown in Fig. S4, the characteristic absorbance slightly decreased with the prolonging of the reaction time due to the adsorption ability of PDA. A similar curve was exhibited when the Sand@COF was applied as the catalyst (Fig. S5). These results suggested the effective catalytic ability of Ag NPs. Furthermore, Ag NPs supported by polydopamine modified sand were used as controls. As shown in Fig. S6, although the characteristic absorbance vanished within $10 \mathrm{~min}$, the content of Ag NPs was $0.30 \mathrm{wt}$ \%, which was much higher than that of our prepared COF-Ag NPs@Sand catalyst $(0.11 \mathrm{wt} \%)$. Furthermore, the turnover frequency (TOF) was applied to exhibit the true catalytic activity of samples, by which the value of COF-Ag NPs@Sand catalyst was calculated as $13.5 \mathrm{~h}^{-1}$, which was much higher than that of Sand-Ag NPs catalyst $\left(5.1 \mathrm{~h}^{-1}\right)$, reflecting the important role of COF as a substrate for catalytic reduction of 4-NP. Besides, the catalytic performances of COF-Ag NPs@Sand catalyst towards other hazardous organic pollutants such as-MB and $\mathrm{CR}$ were measured to demonstrate the university of our prepared catalyst. Fig. 3b illustrated that the characteristic absorbance of CR at $510 \mathrm{~nm}$ decreased within $12 \mathrm{~min}$, indicating the rapid reaction of $\mathrm{CR}$ upon the COF-Ag NPs@Sand catalyst and $\mathrm{NaBH}_{4}$. Similarly, the color of MB faded within 9 min after reducing by COF-Ag NPs@Sand catalyst (Fig. 3c). In our experiments, the reduction reagent $\mathrm{NaBH}_{4}$ was excessive, thus the kinetic equation (1) can be used to evaluate the catalytic activity of the prepared COF-Ag NPs@Sand catalyst. As shown in Fig. 3d, for three organic pollutants, pseudo-first-order reaction kinetics with a linear relationship between $\ln \left(C_{\mathrm{t}} / C_{0}\right)$ and $\mathrm{t}$ could be observed. The slope values of $K_{\text {app }}$ could be calculated to be $0.41 \mathrm{~min}^{-1}, 0.15 \mathrm{~min}^{-1}$, and $0.44 \mathrm{~min}^{-1}$ for 4-NP, CR, and MB, respectively. Moreover, COF-Ag NPs@Sand catalyst loading different Ag NPs was compared. As shown in Fig. S7 and table S1, the catalyst containing $0.09 \mathrm{wt} \%, 0.11 \mathrm{wt} \%$, and $0.15 \mathrm{wt} \% \mathrm{Ag}$ NPs exhibited a similar TOF and the value of $K_{\text {app }}$ increased with Ag NPs content. All these results revealed that the prepared COF-Ag 
a
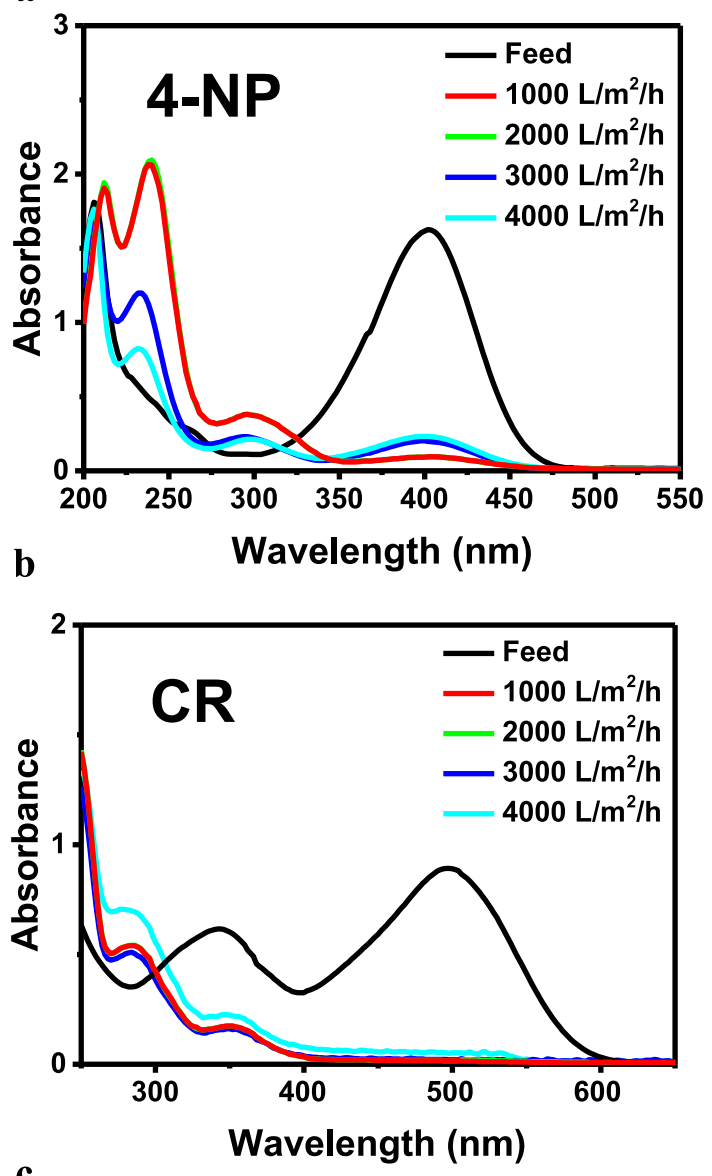

c

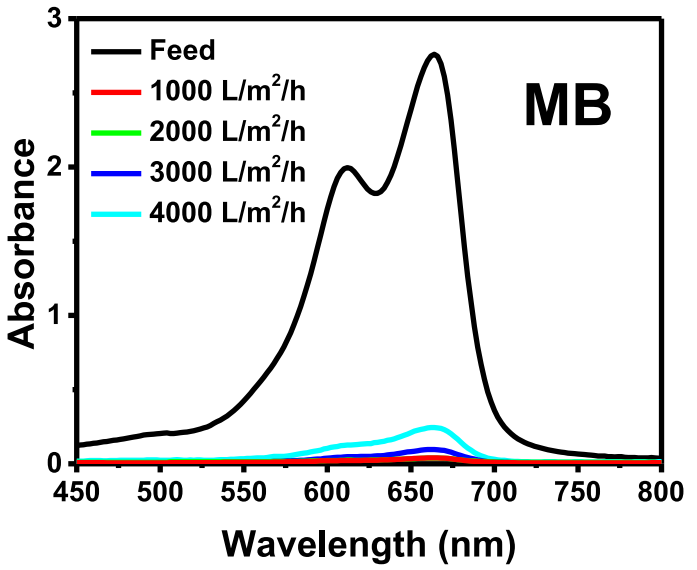

Fig. 5. UV-vis spectra and reduction efficiency of the (a) 4-NP,

NPs@Sand catalyst exhibited a highly effective and universal catalytic activity.

\subsection{Recyclability examination}

The recyclability of the prepared COF-Ag NPs@Sand catalyst was examined by the five successive cycles experiment. For one cycle, the mixture was poured out and the fresh mixture containing organic pollutants and $\mathrm{NaBH}_{4}$ was added. As shown in Fig. 4a, the value of $K_{\text {app }}$ was almost unaltered and only decreased by less than $5 \%$. This result is mainly due to the stability of the prepared COF-Ag NPs@Sand catalyst and the fast-self-sedimentary property of sand-based catalyst in the mixture, avoiding the weight loss of the catalyst during the recyclability
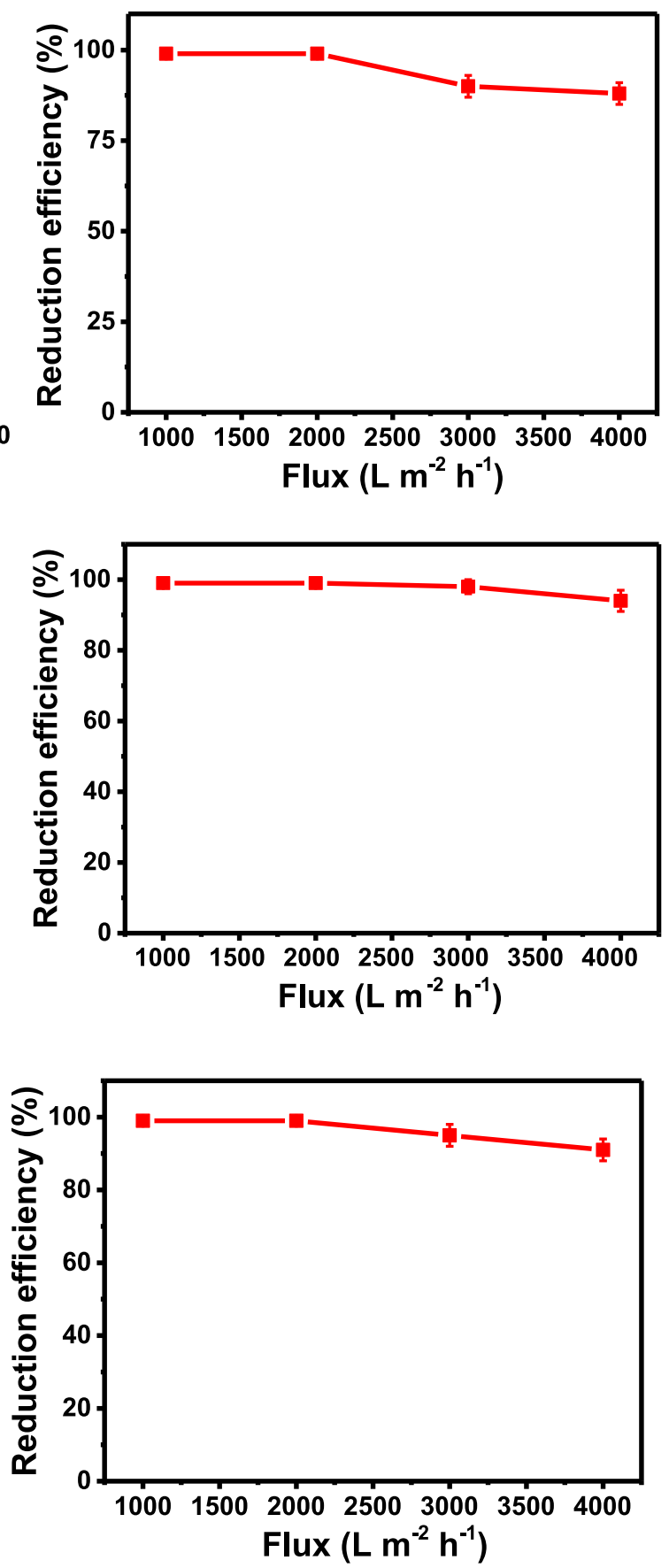

(b) $\mathrm{CR}$, and (c) $\mathrm{MB}$ under flow-through catalytic process at $\mathrm{pH}=7$.

process [29]. Furthermore, COF-Ag NPs without sand was used as a catalyst for control. Although the COF-Ag NPs exhibited a similar TOF of $13.3 \mathrm{~h}^{-1}$ and the value of $K_{\text {app }}$ of $0.38 \mathrm{~min}^{-1}$ compared with COF-Ag NPs@Sand catalyst for one cycle (Fig. S8), the value of $K_{\text {app }}$ decreased by more than $20 \%$ after 5 cycles even under high-speed centrifuge due to the lower density of COF. These results well demonstrated the important role of sand as the substrate for the recyclability of the catalyst. Nevertheless, the recyclability process is inefficient. The pouring out and adding the mixture process spent many times, decreasing the treatment efficiency towards organic pollutants. Hence, a continuous catalytic process is expected. 
$\mathbf{a}$

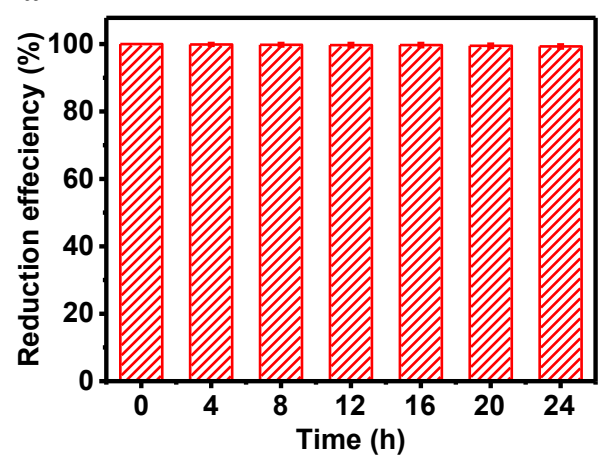

b

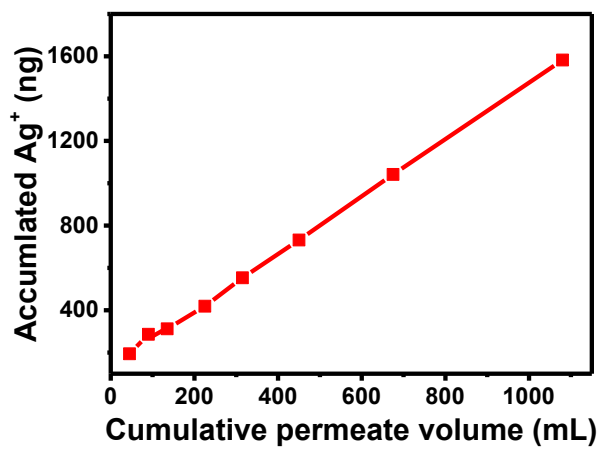

Fig. 6. (a) The reduction efficiency with the prolonging reaction time under flow-through process. (b) the accumulated release of Ag ions from COF-Ag NPs@Sand catalyst under a flux of $1000 \mathrm{LMH}$.

\subsection{Flow-through catalytic process of COF-Ag NPs@Sand}

Considering the advantage of our fabricated COF-Ag NPs@Sand catalyst, a continuous flow-through catalytic setup was designed and applied for the fast-catalytic reduction of organic pollutants (Fig. S9). Typically, 400 mg COF-Ag NPs@Sand catalyst was added in a PVC tube. A feed solution containing organic pollutants was pumped through with the changing fluxes. As shown in Fig. 5a, the absorbance value of 4-NP decreased to near zero as the feed solution passed through the tube containing the COF-Ag NPs@Sand catalyst under the flux of $2000 \mathrm{~L}$ $\mathrm{m}^{-2} \mathrm{~h}^{-1}$ (LMH). The reduction efficiency of the catalyst towards 4-NP was maintained at more than $99 \%$ under the flux of 2000 LMH and decreased to $90 \pm 3 \%$ as the flux up to $3000 \mathrm{LMH}$. The reduction efficiency was further decreased to $88 \pm 3 \%$ when the flux was increased to $4000 \mathrm{LMH}$. The increased flux would decrease the residence and reaction time for the 4-NP and catalyst, thus decreasing the reduction efficiency [30]. That is, the catalytic performance can be further optimized through tailoring the concentration of the COF-Ag NPs@Sand catalyst. Furthermore, the influence of $\mathrm{pH}$ on the catalytic performance was investigated. As shown in Fig. S10, the catalytic performance was unaltered under different $\mathrm{pH}$ values, reflecting the good stability of our prepared catalyst. Besides, the flow-through catalytic performance of the prepared COF-Ag NPs@Sand catalyst was evaluated by other pollutants such as $\mathrm{CR}$ and MB. Fig. $5 \mathbf{b}$ and $5 \mathbf{c}$ presented that the characteristic absorbance of $\mathrm{CR}$ at $510 \mathrm{~nm}$ and $\mathrm{MB}$ at $665 \mathrm{~nm}$ rapidly disappeared under the flux of $2000 \mathrm{LMH}$. Table S2 listed the recently reported catalysts for the reduction of 4-NP, CR, and MB. Our prepared
COF-Ag NPs@Sand catalyst presented better results than these reported. For example, in our previous work, SCOF@Ag NPs with Ag NPs size of ac. $20 \mathrm{~nm}$ and loading of $0.95 \mathrm{wt} \%$ were synthesized. More than 4 times Ag contents were needed to obtain a similar catalytic performance [6]. The good performance of our prepared catalyst can be attributed to the large specific surface areas for supporting abundant small sized Ag NPs and loosely stacked pore structures for the fast passing through the feed solution and continuous catalytic reduction of organic pollutants.

\subsection{The stability of COF-Ag NPs@Sand catalyst}

The stability of the prepared COF-Ag NPs@Sand catalyst was examined. ICP-MS was applied to measure the release of Ag ions. The reduction efficiency was almost unaltered and maintained at more than 99\% after testing for continuous $24 \mathrm{~h}$ (Fig. 6a). A linear relationship between the release of $\mathrm{Ag}$ ions in the filtrate and the permeate volume could be observed from the Fig. 6 b. Only $1.5 \mathrm{ppb}$ Ag ions was detected after testing for $24 \mathrm{~h}$, which was far below the limit in drinking water (100 ppb) [6]. The cumulative Ag ions after testing for $24 \mathrm{~h}$ in the filtrate was only $1.6 \mu \mathrm{g}$, accounting for $0.34 \mathrm{wt} \%$ of the whole content of Ag NPs on the fabricated catalyst. That is, $99.66 \mathrm{wt} \% \mathrm{Ag}$ ions were stably immobilized on the surface of the catalyst. From the image of COF-Ag NPs@Sand catalyst after catalysis for 24 h, the morphology was still kept unchanging (Fig. S11). The XRD curve for COF-Ag NPs@Sand under flow-through experiments after $24 \mathrm{~h}$ further reflected the stability of our prepared catalyst (Fig. S12). The good stability of the catalyst could be contributed to the strong adhesion of polydopamine [31].

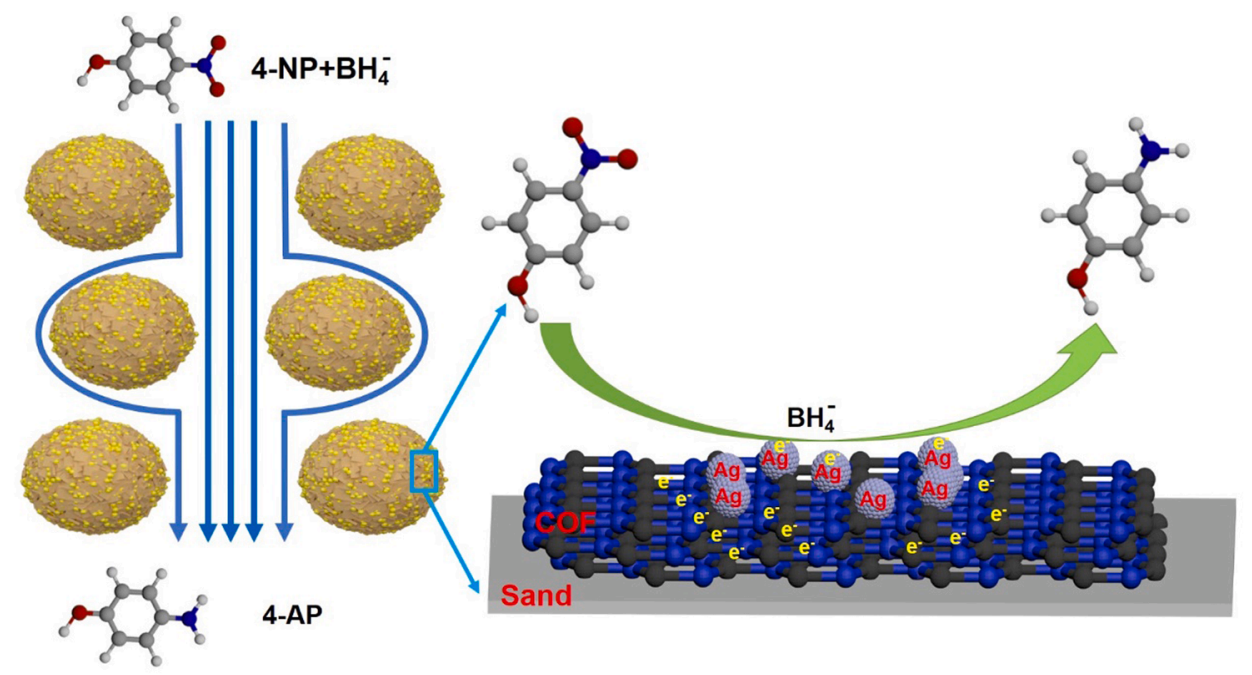

Scheme 2. Catalytic mechanism proposed for explaining the good catalytic performance of our prepared COF-Ag NPs@Sand catalyst. 
Besides, the large-sized sand can effectively prevent the release of catalysts on the filtrate.

\subsection{The catalytic reduction mechanism}

A mechanism was proposed for explaining the good catalytic performance of our prepared COF-Ag NPs@Sand catalyst (Scheme 2). The unique design of the catalyst plays a critical role. First, COF with a good electron transfer ability plays as a cocatalyst and provides more activity sites for catalysis [6]. Second, the substrate sand with good hydrophilicity and loosely stacked pore structure is in favor of fast passing through the aqueous solution [29]. Third, COF-Ag NPs can be immobilized on the sand through polydopamine chemistry [24,32]. Besides, the large size of substrates sand can effectively avoid the leaching out of catalyst during the flow-through catalytic process.

\section{Conclusions}

In conclusion, covalent organic framework/silver nanoparticles (COF-Ag NPs) were designed to immobilize on the polydopamine modified sand to fabricate a COF-Ag NPs@sand heterostructure. A continuous flow-through process towards three model organic pollutants, 4-nitrophenol, Congo red, and methylene blue were carried out and good catalytic performances with the flux of $2000 \mathrm{~L} \mathrm{~m}^{-2} \mathrm{~h}^{-1}$ and more than $99 \%$ reduction efficiency were obtained. Furthermore, good stability and sustainable catalytic ability were exhibited. The good catalytic performance of our prepared catalyst can be attributed to the advantages of sand to immobilize supporting COF-Ag NPs catalysts. Our strategy for the design of a COF-Ag NPs based catalyst using natural material as the substrate can extend to other functional catalysts for organic pollutants treatment.

\section{CRediT authorship contribution statement}

Fei Pan: Writing - original draft. Fengyan Xiao: Writing - review \& editing. Ning Wang: Writing - review \& editing, Resources.

\section{Declaration of Competing Interest}

The authors declare that they have no known competing financial interests or personal relationships that could have appeared to influence the work reported in this paper.

\section{Acknowledgments}

The authors gratefully acknowledge the funding support from National Natural Science Foundation of China (No. 51903247), Key Science and Technology Program of Yantai City (No. 2018ZHGY075), and Shandong Key Laboratory of Coastal Environmental Processes, YICCAS (Grant No. 2019SDHADKFJJ15).

\section{Appendix A. Supplementary material}

Supplementary data to this article can be found online at https://doi. org/10.1016/j.apsusc.2021.150580.

\section{References}

[1] R.K. Sharma, P. Yadav, M. Yadav, R. Gupta, P. Rana, A. Srivastava, R. Zbořil, R. S. Varma, M. Antonietti, M.B. Gawande, Recent development of covalent organic frameworks (COFs): synthesis and catalytic (organic-electro-photo) applications, Mater. Horiz. 7 (2020) 411-454.

[2] P.M. Heintz, B.P. Schumacher, M. Chen, W. Huang, L.M. Stanley, A Pd(II)functionalized covalent organic framework for catalytic conjugate additions of arylboronic acids to $\beta, \beta$-disubstituted enones, Chem. Cat. Chem. 11 (2019) 4286-4290.
[3] W. Gong, Q. Wu, G. Jiang, G. Li, Ultrafine silver nanoparticles supported on a covalent carbazole framework as high-efficiency nanocatalysts for nitrophenol reduction, J. Mater. Chem. A 7 (2019) 13449-13454.

[4] J. Wang, S. Zhuang, Covalent organic frameworks (COFs) for environmental applications, Coord. Chem. Rev, 400 (2019) 213046.

[5] F. Wang, F. Pan, S. Yu, D. Pan, P. Zhang, N. Wang, Towards mass production of a spherical montmorillonite@covalent organic framework@gold nanoparticles heterostructure as a high-efficiency catalyst for reduction of methylene blue, Appl. Clay Sci. 203 (2021) 106007.

[6] N. Wang, F. Wang, F. Pan, S. Yu, D. Pan, Highly efficient silver catalyst supported by a spherical covalent organic framework for the continuous reduction of 4nitrophenol, ACS Appl. Mater. Inter. 13 (2021) 3209-3220.

[7] P. Pachfule, S. Kandambeth, D. Díaz Díaz, R. Banerjee, Highly stable covalent organic framework-Au nanoparticles hybrids for enhanced activity for nitrophenol reduction, Chem. Commun. 50 (2014) 3169-3172.

[8] Y. Zhong, S. Mahmud, Z. He, Y. Yang, Z. Zhang, F. Guo, Z. Chen, Z. Xiong, Y. Zhao, Graphene oxide modified membrane for highly efficient wastewater treatment by dynamic combination of nanofiltration and catalysis, J. Hazard. Mater. 397 (2020) 122774.

[9] N. Wang, F. Xiao, J. Zhang, H. Zhou, Y. Qin, D. Pan, Spherical montmorillonitesupported nano-silver as a self-sedimentary catalyst for methylene blue removal, Appl. Clay Sci. 174 (2019) 146-151.

[10] Y. Wang, L. He, Y. Li, L. Jing, J. Wang, X. Li, Ag NPs supported on the magnetic Al$\mathrm{MOF} / \mathrm{PDA}$ as nanocatalyst for the removal of organic pollutants in water, J. Alloys Compd. 828 (2020) 154340.

[11] A.N. Chishti, F. Guo, A. Aftab, Z. Ma, Y. Liu, M. Chen, J. Gautam, C. Chen, L. Ni, G. Diao, Synthesis of silver doped $\mathrm{Fe}_{3} \mathrm{O}_{4} / \mathrm{C}$ nanoparticles and its catalytic activities for the degradation and reduction of methylene blue and 4-nitrophenol, Appl. Surf. Sci. 546 (2021) 149070.

[12] Y. Chen, Y. Li, L. Dai, G. Qin, J. Guo, Q. Zhang, S. Li, T.A. Sherazi, S. Zhang, Highefficiency Pd nanoparticles loaded porous organic polymers membrane catalytic reactors, Chem. Commun. 57 (2021) 3131-3134.

[13] S. Varshney, R. Bar-Ziv, T. Zidki, On the remarkable performance of silver-based alloy nanoparticles in 4-nitrophenol catalytic reduction, Chem. Cat. Chem. 12 (2020) 4680-4688.

[14] M. Nasrollahzadeh, R. Akbari, Z. Issaabadi, S.M. Sajadi, Biosynthesis and characterization of $\mathrm{Ag} / \mathrm{MgO}$ nanocomposite and its catalytic performance in the rapid treatment of environmental contaminants, Ceram. Int. 46 (2020) 2093-2101.

[15] Z. Gong, T. Ma, F. Liang, Syntheses of magnetic blackberry-like Ni@Cu@Pd nanoparticles for efficient catalytic reduction of organic pollutants, J. Alloys Compd. 873 (2021) 159802.

[16] X. Zhang, L. Chen, Y. Liu, Y. Wang, Q. Duan, Co75Pt25 alloy nanoparticles: A class of catalyst for the catalytic reduction of 4-nitrophenol with enhanced activity and recycling, J. Alloys Compd. 858 (2021) 157700.

[17] T. Bian, J. Zhang, Z. Wang, Z. Wang, L. Liu, J. Meng, J. Zhao, Q. Cai, H. Wang, $\mathrm{MoS}_{2}$-induced hollow $\mathrm{Cu}_{2} \mathrm{O}$ spheres: Synthesis and efficient catalytic performance in the reduction of 4-nitrophenol by $\mathrm{NaBH}_{4}$, Appl. Surf. Sci. 539 (2021) 148285.

[18] Y. Fu, P. Xu, D. Huang, G. Zeng, C. Lai, L. Qin, B. Li, J. He, H. Yi, M. Cheng, C. Zhang, Au nanoparticles decorated on activated coke via a facile preparation for efficient catalytic reduction of nitrophenols and azo dyes, Appl. Surf. Sci. 473 (2019) 578-588.

[19] S. Liu, Y. Qi, L. Cui, Q. Dai, S. Zeng, C. Bai, Controllable synthesis of silver anchored N-doped yolk-shell carbon@ $\mathrm{mSiO}_{2}$ spheres and their application for the catalytic reduction of 4-nitrophenol, Appl. Surf. Sci. 493 (2019) 1013-1020.

[20] Q. Sun, B. Aguila, J. Perman, L.D. Earl, C.W. Abney, Y. Cheng, H. Wei, N. Nguyen, L. Wojtas, S. Ma, Postsynthetically modified covalent organic frameworks for efficient and effective mercury removal, J. Am. Chem. Soc. 139 (2017) 2786-2793.

[21] W. Ma, Q. Zheng, Y. He, G. Li, W. Guo, Z. Lin, L. Zhang, Size-controllable synthesis of uniform spherical covalent organic frameworks at room temperature for highly efficient and selective enrichment of hydrophobic peptides, J. Am. Chem. Soc. 141 (2019) 18271-18277.

[22] Y. Xu, X. Shi, R. Hua, R. Zhang, Y. Yao, B. Zhao, T. Liu, J. Zheng, G. Lu, Remarkably catalytic activity in reduction of 4-nitrophenol and methylene blue by $\mathrm{Fe}_{3} \mathrm{O}_{4} @ \mathrm{COF}$ supported noble metal nanoparticles, Appl. Catal., B 260 (2020) 118142.

[23] Y. Deng, Z. Zhang, P. Du, X. Ning, Y. Wang, D. Zhang, J. Liu, S. Zhang, X. Lu, Embedding ultrasmall Au clusters into the pores of a covalent organic framework for enhanced photostability and photocatalytic performance, Angew. Chem. Int. Ed. 59 (2020) 6082-6089.

[24] N. Wang, Y. Hu, Z. Zhang, Sustainable catalytic properties of silver nanoparticles supported montmorillonite for highly efficient recyclable reduction of methylene blue, Appl. Clay Sci. 150 (2017) 47-55.

[25] L. Wang, H. Xu, Y. Qiu, X. Liu, W. Huang, N. Yan, Z. Qu, Utilization of Ag nanoparticles anchored in covalent organic frameworks for mercury removal from acidic waste water, J. Hazard. Mater. 389 (2020) 121824.

[26] F. Xiao, Y. Qin, N. Wang, D. Pan, Towards mass production of Au nanoparticles supported on montmorillonite microspheres for catalytic reduction of 4nitrophenol, Appl. Clay Sci. 166 (2018) 74-79.

[27] T. Liu, Z. Cui, Y. Liu, X. Bai, In-situ fabrication of ultrafine Pd supported on nitrogen-doped reduced graphene oxide via nitrogen glow discharge plasma for catalytic reduction of 4-Nitrophenol, Appl. Catal. A-Gen. 588 (2019) 117278.

[28] H. Wang, N. Wang, F. Wang, F. Xiao, D. Pan, Spherical montmorillonite-supported molybdenum disulfide nanosheets as a self-sedimentary catalyst for organic pollutants removal, Sep. Purif. Technol. 117346 (2020).

[29] N. Wang, Z. Zhang, J. Huang, Y. Hu, Facile synthesis of copper ions chelated sand via dopamine chemistry for recyclable and sustainable catalysis, Chem. Eng. Sci. 203 (2019) 312-320. 
[30] L. Qi, K. Zhang, W. Qin, Y. Hu, Highly efficient flow-through catalytic reduction of methylene blue using silver nanoparticles functionalized cotton, Chem. Eng. J. 388 (2020) 124252.

[31] P. Zhang, F. Wang, Y. Qin, N. Wang, Exfoliated graphitic carbon nitride nanosheets/gold nanoparticles/spherical montmorillonite ternary porous heterostructures for the degradation of organic dyes, ACS Appl. Nano Mater. 3 (2020) 7847-7857.

[32] Y. Yang, W. Zhu, B. Shi, C. Lü, Construction of a thermo-responsive polymer brush decorated $\mathrm{Fe}_{3} \mathrm{O}_{4} @$ catechol-formaldehyde resin core-shell nanosphere stabilized carbon dots/PdNP nanohybrid and its application as an efficient catalyst, J. Mater. Chem. A 8 (2020) 4017-4029. 